\title{
The Effect of Magnetization Defects on DW Motion in Ferromagnetic Nanowires
}

\author{
Kyle Kimminau, and Andrew Kunz
}

\author{
Physics Department, Marquette University, Milwaukee, WI
}

\begin{abstract}
The propagation of domain walls in ferromagnetic nanowires offers the possibility of ultrafast logic devices and high density data storage. The understanding and control of domain wall motion has become more desirable recently as these devices come closer to realization. Domain walls can only be propagated by small magnetic fields or their movement becomes hindered by the precessional motion of the magnetic dipoles, also called the Walker breakdown effect ${ }^{1}$. Through the use of Landau-Lifshitz Gilbert Simulation, we report a method of eliminating the Walker breakdown. This investigation also offers some insight into how these proposed devices might perform in the high energy radiation of space for extended periods of time.
\end{abstract}

\section{Introduction}

In a ferromagnetic nanowire, it is energetically favorable for the magnetization to be aligned with the length of the wire. Thus, the magnetization lies along the long axis of the wire, with magnetic domains oriented to the left or to the right; the transition region that must exist between these regions is termed a domain wall. The domain wall can be oriented upwards or downwards, and the domains surrounding the domain wall can be oriented head to head or tail to tail. These domain walls can be propagated along the length of the wire by applying an external magnetic field on the sample; a domain with magnetization aligned with the external field will grow at the expense of adjacent domains.

This motion along the wire is smooth and fast for small fields, but fields applied above a certain value, the Walker field limit, cause the dipoles in the domain wall to precess around direction of the external field. This precessional motion, called the Walker breakdown, causes an anti-vortex to nucleate on the edge of the domain wall, and travel across the width of the wire. During this nucleation and movement of the anti-vortex the domain wall itself remains relatively stationary. Following the ejection of the anti-vortex, the wall continues moving smoothly until the next nucleation occurs, creating a periodic behavior and significantly reducing the average speed.

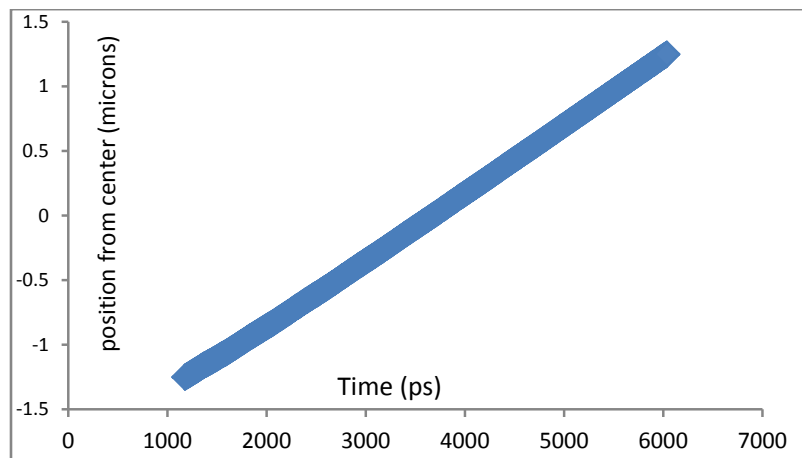

Figure 1: The domain wall is pushed through the wire with an applied field of 15 Oersteds. This causes the wall to move smoothly and quickly along the wire.

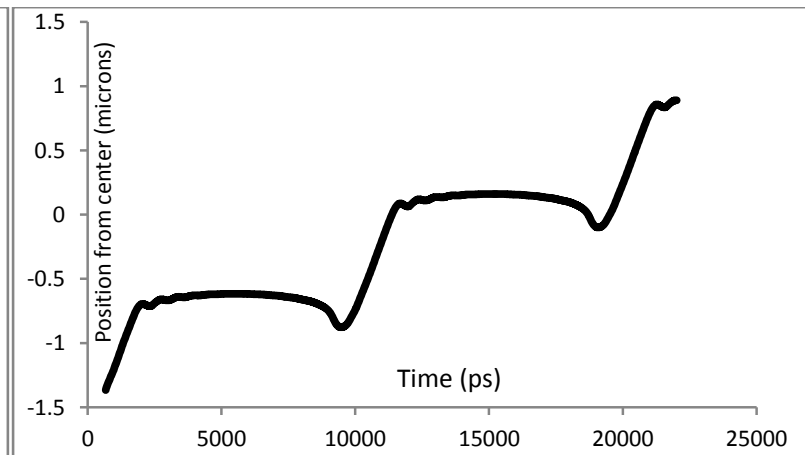

Figure 2: The domain wall is propagated with a field of 30 Oersteds. Instead of smooth fast motion along the wire the motion is periodic and the average speed is very much lower than at fields lower than the Walker limit. 


\section{Micromagnetic Simulation}

The LLG Micromagnetic simulator utilizes the Landau-Lifshitz Gilbert equation of motion for magnetic dipoles in order to simulate interactions within magnetic materials ${ }^{2}$. The equation is

$$
\frac{\partial \vec{m}_{i}}{\partial t}=-\gamma\left(\vec{m}_{i} \times \vec{H}_{i}\right)-\frac{\alpha \gamma}{M_{s}} \vec{m}_{i} \times\left(\vec{m}_{i} \times \vec{H}_{i}\right)
$$

such that $\gamma$ is the gyromagnetic ratio and $H$ is the sum of the applied external field, the dipole field and an exchange field. The first term on the right hand side of the equation describes how the dipoles precess about the magnetic field. This term is responsible for the Walker breakdown behavior observed at high fields. The second term describes how the dipoles align with the magnetic field, and is why the application of an external magnetic field propagates the domain wall through the wire. At low fields the first term is dominated by the second term, so Walker breakdown does not occur.

The material parameters used in the trials model permalloy with no crystalline anisotropy: $\alpha=.008$, exchange stiffness $A=1.3 \times 10^{-11} \mathrm{~J} / \mathrm{m}$, and the saturation magnetization of the material $\mathrm{M}_{\mathrm{s}}=8 \times 10^{5} \mathrm{~A} / \mathrm{m}$. The magnetic material is dicretized by the program into small blocks of magnetization that act as the dipoles in the system and are usually within 1-10 nm on each side. The blocks used in our trials were $2 \mathrm{~nm}$ on a side. The program then calculates the motion of every dipole initially and repeats the calculation at time increments, or steps, that are less than a picosecond $^{2}$. The high spatial and temporal resolutions together allow for very detailed investigation into the important phenomena that occur in magnetic interactions.

\section{Methods}

The saturation magnetization has been found to be diminished in magnetic materials if irradiated with certain particles ${ }^{4}$. In order to model the effects of high energy particles colliding with the magnetized sample, we used the program to eliminate random blocks of magnetization in the wire. The density of defects was gradually increased from $0 \%$ to $10 \%$ density in increments of $.5 \%$, and then from $10 \%$ to $25 \%$ in increments of $2.5 \%$. These defect densities were saved as masks and used for trials over a variation of applied magnetic fields.

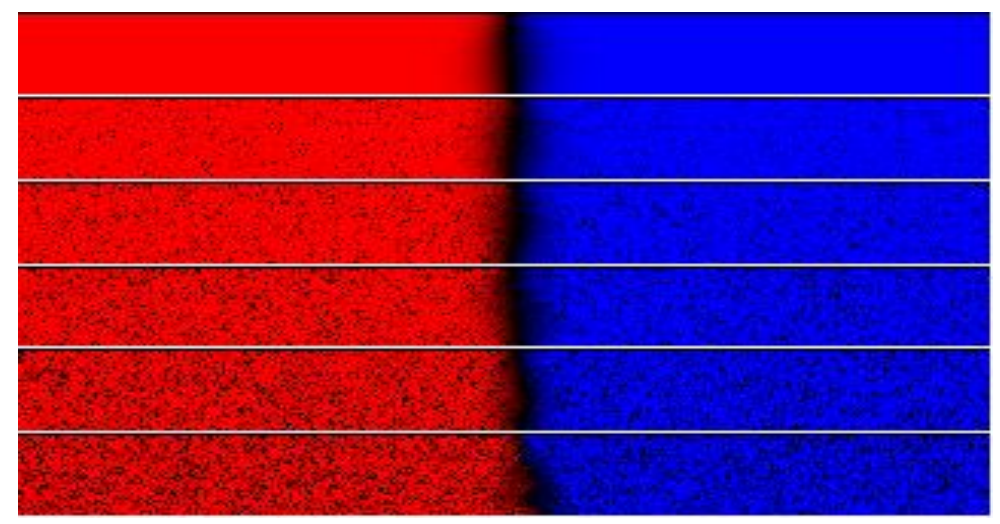

Figure 3: The series of wires have had blocks of magnetization eliminated to model defects from irradiation. The defect densities, from top to bottom, are as follows: $0 \%, 5 \%, 10 \%, 15 \%, 20 \%$, and $25 \%$. The wire dimensions used were $5 \mu \mathrm{mx} 100 \mathrm{nmx} 2 \mathrm{~nm}$. 


\section{Results}

The addition of magnetization defects to the nanowires has two significant effects on domain wall motion. The first effect is an overall decrease in domain wall speed, whether the applied field is below or above the Walker field. When a domain wall is propagated by a field less than the Walker limit the wall speed decreases with increasing defect density, as might be expected. No considerable change in speed occurs until the defect density reaches $10 \%$. The speed of the wall is only lessened by $10 \%$ at $10 \%$ defect density. However between $10 \%$ and $20 \%$, the speed of domain wall pushed by fields less than the Walker limit is halved if it is not stopped altogether. The same effect occurs when the wall is moved with fields greater than the Walker limit, but the speed doesn't begin to decrease until about $10 \%$ density. Regardless of field, no domain wall can be propagated in a defect density of $25 \%$ or greater.

The second effect observed due to the defects in the magnetization is the elimination of the Walker breakdown effect. In the presence of defects, the motion of a domain wall pushed by fields above the Walker limit becomes more smooth and linear. This effect becomes more prominent as the defect density increases. The domain wall speed reaches its' max at about $10 \%$ defect density, and is where the first effect starts to overpower the propagation. At low densities the domain wall continues to nucleate an anti-vortex, and clusters of defects large enough act as pinning sites for these anti-vortices. The Walker breakdown stems from the cross product of the magnetic field, and the moments of the dipoles in the domain wall. If the magnetization were to be perturbed inside the domain wall geometry, the cross product would diminish, causing the first term in the equation of motion to be overpowered. The defects provide these perturbations by forcing the magnetization to form around the area lacking magnetization, thus countering the breakdown effect.

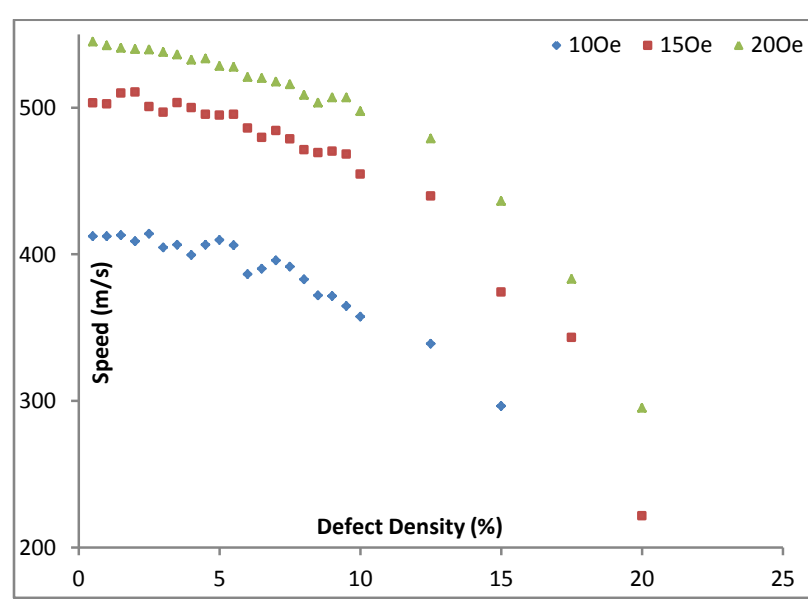

Figure 4: Domain walls with increasing defect densities are propagated with three fields under the Walker field. It can be seen that speeds do not decrease significantly until after $10 \%$ defect density.

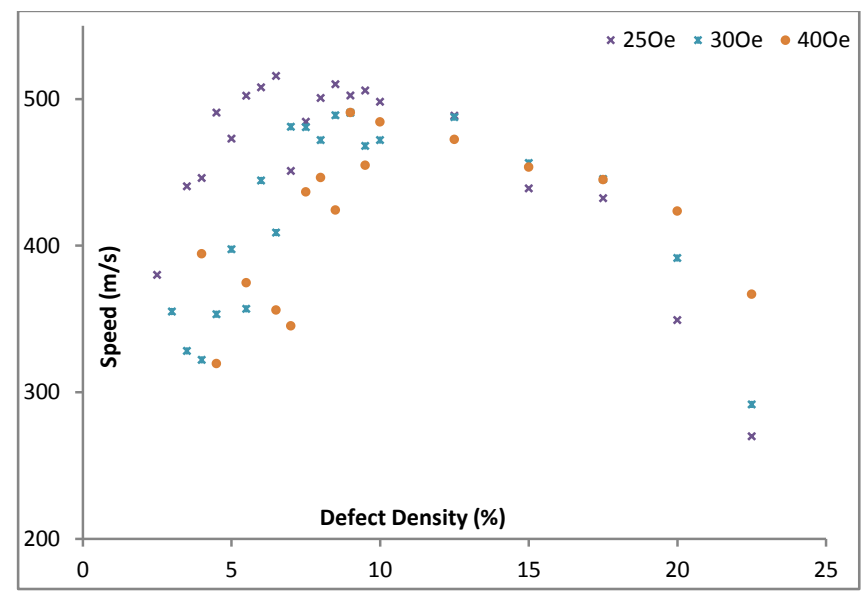

Figure 5: The walls in this graph were moved with three fields above the Walker limit. The wall speed increases with increasing fill until about $10 \%$ density for all fields. After $10 \%$ the walls act similarly to walls pushed by fields less than the Walker limit 


\section{Conclusions}

The proposed magnetic nanowire devices utilize the control of domain walls to store/analyze information. In order to move and control these walls a magnetic field must be applied, but these fields must be under the Walker field limit. Generating such small and precise magnetic fields would prove difficult if not impossible. Therefore the elimination of the Walker breakdown is of importance if the computers of the future will include magnetic devices. We have found that the Walker breakdown is countered if random defects are placed in the wires. However, pinning occurs when an anti-vortex comes in contact with a cluster of defects. A controlled pattern of defects applied during production of the nanowires could eliminate the pinning sites and serve to eliminate the Walker breakdown more effectively.

We have also shown that domain wall speed is not dramatically changed for small defect densities. This could mean that these devices are plausible for use in space, but unfortunately they will inevitably require replacements. The current electronic devices do not face this issue, and could be more desirable even if the magnetic devices are operational under small defect densities, especially considering pinning issues with the lower densities.

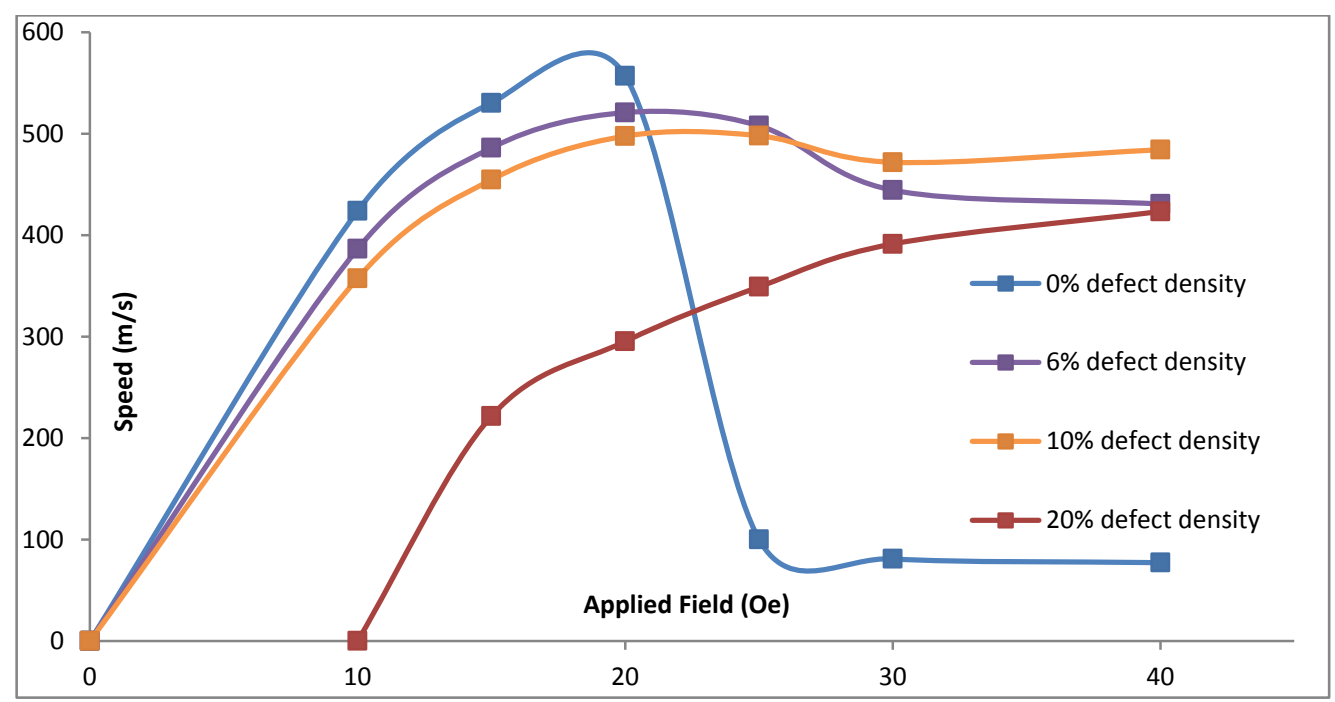

Figure 6: The behavior of domain walls in nanowires with no defects is shown by the blue data; the Walker breakdown occurs just above 20 Oe and the speed decreases significantly. The other sets of data show various defect densities and how the breakdown affects the motion of the walls. The $10 \%$ defect density gives the highest speed at higher fields, and at higher densities the wall speed is diminished due to the number of perturbation. 


\section{Future Projects}

Defect clusters have been observed to act as pinning sites for anti-vortices. This is of interest if we are to prevent the pinning of domain walls at undesired locations. The energy well created by the defects can most likely be described using a simple magnetic charge model. Magnetic charge is defined as

$$
Q_{m}=-\mu_{0} \oint_{S} \vec{M} \cdot d \vec{S}
$$

where $M$ is the magnetization. By using the LLG simulator we can calculate the charge in each block and map out the charge geometry. By doing this we can investigate the energies of the domain wall and any type of defect that it might interact with. This might lead to defect patterns better suited for eliminating breakdown behavior and avoiding pinning of domain walls.

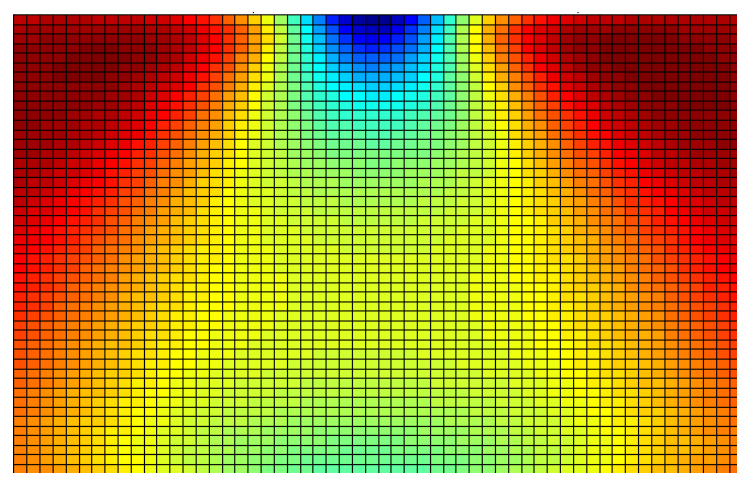

Figure 7: The charge structure of a head to head down domain wall. It seems that the densest area of charge is located at the top of its triangle geometry.

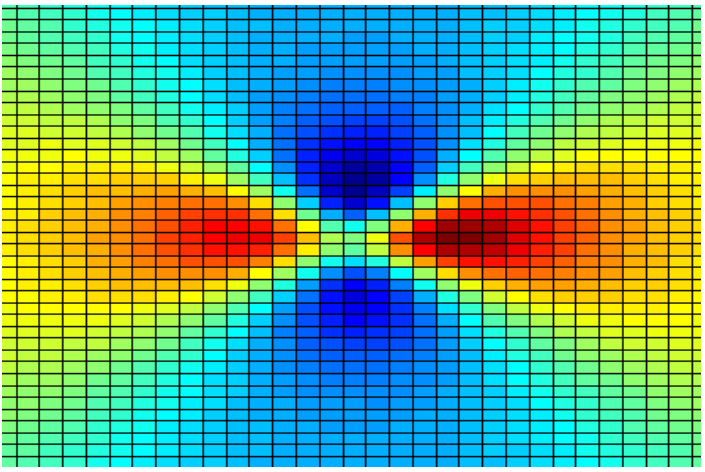

Figure 8: Above is the charge structure for an anti-vortex nucleated from a head to head down domain wall (left). The charge density is high around the core of the antivortex. The dynamics could explain the pinning phenomena observed.

\section{References}

[1] N.L. Schryer, L.R. Walker, "The motion of $180^{\circ}$ domain walls in uniform dc magnetic fields", JAP 45, 5406 (1974).

[2] LLG Micromagnetics Simulator, LLG Inc.

[3] J.-Y. Lee, et al., "Dynamic transformation of the internal structure of a moving domain wall in magnetic nanostripes", Phys. Rev. B. 76, 184408 (2007)

[4] A. Vogel, S. Wintz, J. Kimling, M. Bolte, "Domain-Wall Pinning and Depinning at Soft Spots in Magnetic Nanowires", IEEE Trans. Magn. 46, (2010) 